\title{
REAL ANALYTIC BOUNDARY REGULARITY OF THE CAUCHY KERNEL ON CONVEX DOMAINS
}

\author{
SO-CHIN CHEN
}

(Communicated by Clifford J. Earle, Jr.)

\begin{abstract}
It is well-known that in one complex variable the Cauchy integral preserves real analyticity near the boundary. In this paper we show that the same conclusion also holds on convex domains with real analytic boundary in higher dimension, where the Cauchy kernel is given by the Cauchy-Fantappie form of order zero generated by the (1.0)-form $C(\xi, z)$,
\end{abstract}

$$
C(\xi, z)=\left(\sum_{j=1}^{n} \frac{\partial r}{\partial \xi_{j}}(\xi) d \xi_{j}\right)\left(\sum_{j=1}^{n} \frac{\partial r}{\partial \xi_{j}}(\xi)\left(\xi_{j}-z_{j}\right)\right)^{-1},
$$

where $r(\xi)$ is the defining function of the domain.

\section{INTRODUCTION}

In one complex variable there is a classical theorem concerning the real analytic boundary regularity of the Cauchy kernel which states:

Theorem. Let $D$ be a smooth bounded domain in $\mathbf{C}$ with real analytic boundary, and let $z_{0} \in b D$ be a boundary point. Let $U$ be an open neighborhood of $z_{0}$ in C. Suppose that $f(\zeta) \in L^{2}(b D) \cap C^{w}(b D \cap U)$. Then the integral

$$
F(z)=\int_{b D} \frac{f(\zeta)}{\zeta-z} d \zeta, \text { for } z \in D,
$$

defines a holomorphic function on $D$ that is real analytic up to the boundary on $b D \cap U$.

It would be very interesting to generalize this type of theorem to higher dimension. Unfortunately, this cannot be done in general. We do not even know how to write down an appropriate analog of the Cauchy kernel on a smooth bounded domain of holomorphy with real analytic boundary. So in this paper we are going to study this problem on convex domains, where there is a natural

Received by the editors March 15, 1989 .

1980 Mathematics Subject Classification (1985 Revision). Primary 32A25, 32A40.

Key words and phrases. convex domain, Cauchy-Fantappiè form. 
Cauchy kernel as follows: Let $C(\xi, z)$ be the generating $(1,0)$-form given by

$$
C(\xi, z)=\frac{\partial r}{\phi(\xi, z)}=\frac{\sum_{j=1}^{n} \frac{\partial r}{\partial \xi_{j}}(\xi) d \xi_{j}}{\sum_{j=1}^{n} \frac{\partial r}{\partial \xi_{j}}(\xi)\left(\xi_{j}-z_{j}\right)},
$$

the Cauchy kernel $\Omega_{0}(C)$ is then defined to be the Cauchy-Fantappiè form of order zero,

$$
\Omega_{0}(C(\xi, z))=C(\xi, z) \wedge\left(\bar{\partial}_{\xi} C(\xi, z)\right)^{n-1} .
$$

For more details see Range [2].

Here are our main results.

Theorem 1. Let $D \subseteq \mathbf{C}^{n}, n \geq 2$, be a smooth bounded convex domain with real analytic defining function $r(z)$, and let $z_{0} \in b D$ be a boundary point. Let $U$ be a small open neighborhood of $z_{0}$ in $\mathbf{C}^{n}$. Suppose that $f(\xi) \in L^{2}(b D) \cap$ $C^{w}(b D \cap U)$. Then the integral

$$
F(z)=\int_{b D} f(\xi) \Omega_{0}(C(\xi, z)), \quad \text { for } z \in D
$$

defines a holomorphic function on $D$ that is real analytic up to the boundary $b D \cap U$. In particular $F(z)$ can be extended holomorphically across $b D \cap U$.

Corollary 2. Let $D$ be defined as in Theorem 1. Suppose that $f(\xi) \in C^{w}(b D)$. Then $F(z) \in C^{w}(\bar{D})$. In particular $F(z) \in H(\bar{D})$.

We make a remark that the proof we present here for Theorem 1 also works for the case $n=1$, and if $n=1$ the Cauchy kernel $\Omega_{0}(C)$ reduces to the classical one,

$$
\Omega_{0}(C)=\frac{\frac{\partial r}{\partial \xi}(\xi) d \xi}{\frac{\partial r}{\partial \xi}(\xi)(\xi-z)}=\frac{d \xi}{\xi-z}
$$

\section{Proof of TheOReM 1}

Let $D \subseteq \mathbf{C}^{n}, n \geq 2$, be a smooth bounded convex domain with real analytic defining function $r(z)$. The Cauchy kernel on $D$ is defined as follows. For $\xi \in b D$ and $z \in D$ one defines the generating $(1,0)$-form $C(\xi, z)$ by

$$
C(\xi, z)=\frac{\partial r}{\phi(\xi, z)},
$$

where $\partial r(\xi)=\sum_{j=1}^{n} \frac{\partial r}{\partial \xi_{j}}(\xi) d \xi_{j}$ and $\phi(\xi, z)=\sum_{j=1}^{n} \frac{\partial r}{\partial \xi_{j}}(\xi)\left(\xi_{j}-z_{j}\right)$. 
The Cauchy kernel $\Omega_{0}(C)$ for $D$ is then defined to be the CauchyFantappiè form of order zero as follows:

$$
\begin{aligned}
\Omega_{0}(C(\xi, z)) & =C(\xi, z) \wedge\left(\bar{\partial}_{\xi} C(\xi, z)\right)^{n-1} \\
& =\frac{(\partial r) \wedge(\bar{\partial} \partial r)^{n-1}}{\phi^{n}(\xi, z)} \\
& =\frac{\left(\sum_{j=1}^{n} \frac{\partial r}{\partial \xi_{j}} d \xi\right) \wedge\left(\sum_{j, k=1}^{n} \frac{\partial^{2} r}{\partial \bar{\xi}_{k} \partial \xi_{j}} d \bar{\xi}_{k} d \xi_{j}\right)^{n-1}}{\phi^{n}(\xi, z)} \\
& =\sum_{\substack{\text { finite sum } \\
|I|=n-1}} \frac{A_{I}(\xi)}{\phi^{n}(\xi, z)} d \xi_{1} \ldots d \xi_{n} d \bar{\xi}_{I},
\end{aligned}
$$

where $I$ denotes the increasing multiindices of order $n-1$, and $A_{I}(\xi)$ is a polynomial that involves the first and second derivatives of the defining function $r(\xi)$. Hence $A_{I}(\xi)$ is real analytic in some open neighborhood of $\bar{D}$.

The convexity of the domain $D$ implies $\phi(\xi, z) \neq 0$ for $\xi \in b D$ and $z \in D$. Therefore it makes sense to define the following integral for $f(\xi) \in L^{2}(b D)$,

$$
F(z)=\int_{b D} f(\xi) \Omega_{0}(C(\xi, z)) .
$$

Since $\phi(\xi, z)$ is holomorphic in $z$, we see that $F(z) \in H(D)$.

The main purpose of this paper is to show real analytic boundary regularity of this integral. Namely, if $z_{0} \in b D$ is a fixed boundary point and $f(\xi)$ is also real analytic in some open neighborhood of $z_{0}$ in the boundary, then $F(z)$ is also real analytic up to the boundary near $z_{0}$. In particular it follows that $F(z)$ can be extended holomorphically across the boundary near $z_{0}$.

Before we begin to prove Theorem 1, we will state several lemmas concerning the function $\phi(\xi, z)$ that are needed in the sequel. First the following lemma is proved in Range [1].

Lemma 2.2. Let $D$ and $\phi(\xi, z)$ be defined as above, and let $z_{0} \in b D$. Then there exists a positive integer $m_{0}$ and constants $A, \delta_{1}, \delta_{2}>0$ such that

$$
|\phi(\xi, z)| \geq A\left(d(z, b D)+|\operatorname{Im} \phi(\xi, z)|+|\xi-z|^{m_{0}}\right),
$$

for $\xi \in b D \cap B\left(z_{0}, \delta_{1}\right)$ and $z \in \bar{D} \cap B\left(\xi, \delta_{2}\right)$, where $d(z, b D)$ denotes the distance between $z$ and the boundary $b D$.

Lemma 2.4. Let $D$ and $\phi(\xi, z)$ be defined as above, and let $z_{0} \in b D$. For $\delta \ll \min \left(\delta_{1}, \delta_{2}\right)$, we have for some constant $C>0$,

$$
\int_{b D \cap B\left(z_{0}, \delta\right)}|\log \phi(\xi, z)| d S \leq C
$$

uniformly for all $z \in D \cap B\left(z_{0}, \delta\right)$. 
Proof. First by a standard choice (e.g. see Range [2]) one may introduce a local coordinate system $\left(t_{1}, \ldots, t_{2 n}\right)=t=t(\xi, z)$ on $B(z, \eta)$, for some $\eta>0$, such that the following hold.

(i) $t_{1}(\xi, z)=r(\xi)$ and $t(z, z)=(r(z), 0, \ldots, 0)$,

(ii) $t_{2}(\xi, z)=\operatorname{Im} \phi(\xi, z)$,

(iii) $|t(\xi, z)|<1$ for $\xi \in B(z, \eta)$,

(iv) The Jacobian is bounded both from above and from below uniformly for all $z \in \bar{D} \cap B\left(z_{0}, \delta\right)$.

Hence by Lemma 2.2 for sufficiently small $\delta$ we have

$$
\begin{aligned}
|\log \phi(\xi, z)| & \lesssim|\log | \phi(\xi, z)||+|\arg \phi(\xi, z)| \\
& \lesssim|\log A|+|\log | \xi-\left.z\right|^{m_{0}}|+| \arg \phi(\xi, z) \mid .
\end{aligned}
$$

Since we have $\frac{1}{2} \pi \leq \arg \phi(\xi, z) \leq \frac{3}{2} \pi$ for all $z \in D \cap B\left(z_{0}, \delta\right)$ and $\xi \in$ $b D \cap B\left(z_{0}, \delta\right)$, hence by setting $t^{\prime}=\left(t_{2}, \ldots, t_{2 n}\right)$ we obtain

$$
\begin{aligned}
\int_{b D \cap B\left(z_{0}, \delta\right)}|\log \phi(\xi, z)| d S & \lesssim 1+\int_{b D \cap B\left(z_{0}, \delta\right)}|\log | \xi-\left.z\right|^{m_{0}} \mid d S \\
& \lesssim 1+\left.\int_{\left|t^{\prime}\right|<1}|\log | t^{\prime}\right|^{m_{0}} \mid d t_{2} \ldots d t_{2 n} \\
& \lesssim 1+\int_{0}^{1}\left|\log \left(r^{m_{0}}\right)\right| \cdot r^{2 n-2} d r \\
& \lesssim 1 .
\end{aligned}
$$

The last inequality holds because $n \geq 2$. This also completes the proof of the lemma.

Now we are ready to prove our main result. In fact what we are going to prove is a more general setting, namely,

Theorem 2.5. Let $D$ and $\phi(\xi, z)$ be defined as above, and let $z_{0} \in b D$. If $A(\xi) \in C^{w}(b D)$ and $f(\xi) \in L^{2}(b D) \cap C^{w}(b D \cap U)$, where $U$ is an open neighborhood of $z_{0}$ in $\mathbf{C}^{n}$, then for each increasing multiindex $I$ with $|I|=n-1$ and each integer $m \in \mathbf{N}$, the integral

$$
F(z)=\int_{b D} f(\xi) \frac{A(\xi)}{\phi^{m}(\xi, z)} d \xi_{1} \ldots d \xi_{n} d \bar{\xi}_{I}
$$

defines a holomorphic function on $D$ that is real analytic up to the boundary on $b D \cap U$.

Theorem 1 will then follow immediately from Theorem 2.5.

Proof of Theorem 2.5. It is clear that $F(z)$ is holomorphic on $D$. Hence the conclusion will follow by Sobolev's embedding theorem if one can show that the following estimate

$$
\left|\frac{\partial^{\alpha}}{\partial z^{\alpha}} F(z)\right| \leq C C^{|\alpha|} \cdot|\alpha| !,
$$


holds uniformly for all $z \in D \cap$ (some neighborhood of $z_{0}$ in $\mathbf{C}^{n}$ ), and for all multiindices $\alpha$.

To prove (2.7) we first fix two open balls $B_{j}=B\left(z_{0}, \varepsilon_{j}\right), j=1,2$, with $\varepsilon_{1}<\varepsilon_{2}$ and such that $\bar{B}_{2} \subseteq U$. Put $V_{j}=b D \cap B_{j}, j=1,2$. Then by a lemma due to Ehrenpreis there exists a constant $M_{0}>0$ such that for every positive integer $k$ one can find $\phi_{k} \in C_{0}^{\infty}\left(V_{2}\right)$ with $0 \leq \phi_{k} \leq 1, \phi_{k} \equiv 1$ on $V_{1}$ and

$$
\left|D^{\alpha} \phi_{k}\right| \leq M_{0}\left(M_{0} k\right)^{|\alpha|} \text {, for }|\alpha| \leq k
$$

For an outline of the proof of this lemma see Tartakoff [3].

So one can decompose $F(z)$ into two parts, i.e., $F(z)=F_{1}(z)+F_{2}(z)$, where

$$
F_{1}(z)=\int_{b D} \phi_{k}(\xi) f(\xi) \frac{A(\xi)}{\phi^{m}(\xi, z)} d \xi_{1} \ldots d \xi_{n} d \bar{\xi}_{I}
$$

and

$$
F_{2}(z)=\int_{b D}\left(1-\phi_{k}(\xi)\right) f(\xi) \frac{A(\xi)}{\phi^{m}(\xi, z)} d \xi_{1} \ldots d \xi_{n} d \bar{\xi}_{I}
$$

It follows from the estimate (2.3) and the convexity of the domain $D$ that there exists a constant $C_{1}>0$ such that

$$
\left|D_{z}^{\alpha} F_{2}(z)\right| \leq C_{1} C_{1}^{|\alpha|} \cdot|\alpha| !
$$

uniformly for all $z \in D \cap B\left(z_{0}, \varepsilon\right)$ with $0<\varepsilon \ll \varepsilon_{1}$ and all $k$.

The remainder of this paper is devoted to the estimate of $F_{1}(z)$. First we show that-perhaps with a larger constant $M$-the function $\phi_{k}(\xi) f(\xi) A(\xi)$ also satisfies the estimate (2.8).

Lemma 2.9. There exists a constant $M>0$ such that for each multiindex $\alpha$ with $|\alpha| \leq k$, we have

$$
\left|D^{\alpha}\left(\phi_{k}(\xi) f(\xi) A(\xi)\right)\right| \leq M(M k)^{|\alpha|}
$$

Proof of Lemma 2.9. Since $f(\xi) A(\xi)$ is real analytic on $b D \cap U$, there exists a $R>0$ such that for all $\xi \in V_{2}$ we have

$$
\left|D^{\beta}(f(\xi) A(\xi))\right| \leq R R^{|\beta|} \cdot|\beta| !
$$


for all multiindices $\beta$. So

$$
\begin{aligned}
\left|D^{\alpha}\left(\phi_{k}(\xi) f(\xi) A(\xi)\right)\right| \leq & \sum_{\substack{j=0 \\
\alpha_{1}+\alpha_{2}=\alpha \\
\left|\alpha_{1}\right|=j \\
\left|\alpha_{2}\right|=|\alpha|-j}}^{|\alpha|}\left(\begin{array}{c}
|\alpha| \\
j
\end{array}\right)\left|D^{\alpha_{1}} \phi_{k} \| D^{\alpha_{2}}(f(\xi) A(\xi))\right| \\
& \leq \sum_{j=0}^{|\alpha|} \frac{|\alpha| !}{j !(|\alpha|-j) !} \cdot M_{0}\left(M_{0} k\right)^{j} \cdot R R^{|\alpha|-j} \cdot(|\alpha|-j) ! \\
& =M_{0}\left(M_{0} k\right)^{|\alpha|} \cdot \sum_{j=0}^{|\alpha|} \frac{|\alpha| \cdots(j+1)}{k^{|\alpha|-j}} \cdot R \cdot\left(\frac{R}{M_{0}}\right)^{|\alpha|-j} \\
& \leq M_{0}\left(M_{0} k\right)^{|\alpha|} \cdot \sum_{l=0}^{\infty} R \cdot\left(\frac{1}{2}\right)^{l} \\
& \leq\left(2 R M_{0}\right)\left(M_{0} k\right)^{|\alpha|},
\end{aligned}
$$

if we choose $M_{0} \geq 2 R$. So if we let our new $M$ be $\max \left(2 R M_{0}, M_{0}\right)$, we are done. This completes the proof of Lemma 2.9.

Therefore from now on we will call $\phi_{k}(\xi) f(\xi) A(\xi)$ by $g_{k}(\xi)$ such that the estimate (2.10) holds for $g_{k}(\xi)$. Next we consider the operation of $\frac{\partial}{\partial z_{j}}$ on $F_{1}(z)$ for all $z \in D \cap B\left(z_{0}, \varepsilon\right)$.

$$
\begin{aligned}
\frac{\partial}{\partial z_{j}} F_{1}(z) & =\int_{b D} \frac{\partial}{\partial z_{j}}\left(g_{k}(\xi) \cdot \frac{1}{\phi^{m}(\xi, z)}\right) d \xi_{1} \cdots d \xi_{n} d \bar{\xi}_{I} \\
& =\int_{b D} g_{k}(\xi)\left(\frac{m \frac{\partial r}{\partial \xi_{j}}(\xi)}{\phi^{m+1}(\xi, z)}\right) d \xi_{1} \cdots d \xi_{n} d \bar{\xi}_{I}
\end{aligned}
$$

Put $T=\sum_{j=1}^{n} \frac{\partial r}{\partial \xi_{j}} \frac{\partial}{\partial \xi_{j}}-\sum_{j=1}^{n} \frac{\partial r}{\partial \xi_{j}} \frac{\partial}{\partial \xi_{j}}$. We see that $T$ is tangent to the boundary and satisfies

$$
T \phi(\xi, z)=\sum_{j=1}^{n}\left(T\left(\frac{\partial r}{\partial \xi_{j}}\right)\right)\left(\xi_{j}-z_{j}\right)+\sum_{j=1}^{n}\left|\frac{\partial r}{\partial \xi_{j}}\right|^{2} .
$$

It shows that $T \phi(\xi, z)$ does not vanish near the diagonal of $b D \times b D$. So we may assume that $T \phi(\xi, z) \neq 0$ on $U_{z} \times U_{\xi}$, where $U$ is given in Theorem 1 or Theorem 2.5. Let the adjoint $T^{*}$ of $T$ be given by

$$
T^{*}=-T+h(\xi),
$$

where $h(\xi)$ is an analytic function defined on $U$. 
Then by integration by parts $(2.11)$ becomes

$$
\begin{aligned}
\frac{\partial}{\partial z_{j}} F_{1}(z) & =\int_{b D} g_{k}(\xi)\left(-\frac{\frac{\partial r}{\partial \xi_{j}}}{T \phi} T\left(\frac{1}{\phi^{m}(\xi, z)}\right)\right) d \xi_{1} \cdots d \xi_{n} d \bar{\xi}_{I} \\
& =\int_{b D}\left(T^{*}\left(-\frac{\frac{\partial r}{\partial \xi_{j}}}{T \phi} \cdot g_{k}(\xi)\right)\right) \frac{1}{\phi^{m}(\xi, z)} d \xi_{1} \cdots d \xi_{n} d \bar{\xi}_{I} \\
& =\int_{b D}\left(\left(a_{j}(\xi, z) T+b_{j}(\xi, z)\right) g_{k}(\xi)\right) \frac{1}{\phi^{m}(\xi, z)} d \xi_{1} \cdots d \xi_{n} d \bar{\xi}_{I},
\end{aligned}
$$

where $a_{j}(\xi, z)=\left(\frac{\partial r}{\partial \xi_{j}}\right)(T \phi)^{-1}$ and $b_{j}(\xi, z)=T\left(\frac{\partial r}{\partial \xi_{j}}(T \phi)^{-1}\right)-h \cdot\left(\frac{\partial r}{\partial \xi_{j}}\right)(T \phi)^{-1}$. We see that both $a_{j}(\xi, z)$ and $b_{j}(\xi, z)$ are real analytic functions in $\xi$ and $z$ on $U_{z} \times U_{\xi}$ for $j=1, \ldots, n$. Since both $a_{j}(\xi, z)$ and $b_{j}(\xi, z)$ involve variables $\xi$ and $z$, then if we apply another derivative $\frac{\partial}{\partial z_{i}}, i=1, \ldots, n$, we will end up with two terms as follows:

$$
\begin{aligned}
\frac{\partial^{2}}{\partial z_{i} \partial z_{j}} F_{1}(z)= & \frac{\partial}{\partial z_{i}} \int_{b D}\left(\left(a_{j}(\xi, z) T+b_{j}(\xi, z)\right) g_{k}(\xi)\right) \frac{1}{\phi^{m}(\xi, z)} d \xi_{1} \ldots d \xi_{n} d \bar{\xi}_{I} \\
= & \int_{b D}\left(\frac{\partial}{\partial z_{i}}\left(a_{j}(\xi, z) T+b_{j}(\xi, z)\right) g_{k}(\xi)\right) \frac{1}{\phi^{m}(\xi, z)} d \xi_{1} \ldots d \xi_{n} d \bar{\xi}_{I} \\
& +\int_{b D}\left(( a _ { i } ( \xi , z ) T + b _ { i } ( \xi , z ) ) \left(a_{j}(\xi, z) T\right.\right. \\
& \left.\left.+b_{j}(\xi, z)\right) g_{k}(\xi)\right) \frac{1}{\phi^{m}(\xi, z)} d \xi_{1} \ldots d \xi_{n} d \bar{\xi}_{I} .
\end{aligned}
$$

Therefore if we let $Z$ denote any one of the following first order differential operators $\frac{\partial}{\partial z_{j}}$ or $a_{j}(\xi, z) T+b_{j}(\xi, z)$ for $j=1, \ldots, n$, we see that in general $\frac{\partial^{\alpha} F_{1}}{\partial z^{\alpha}}$ is of the following form for any multiindex $\alpha$,

$$
\begin{aligned}
& \frac{\partial^{\alpha}}{\partial z^{\alpha}} F_{1}(z)=\underline{2^{|\alpha|-1}} \int_{b D}\left(Z^{|\alpha|} g_{k}(\xi)\right) \frac{1}{\phi^{m}(\xi, z)} d \xi_{1} \cdots d \xi_{n} d \bar{\xi}_{I} \\
& =\underline{2^{|\alpha|-1}} \cdot \frac{(-1)^{m}}{(m-1) !} \int_{b D}\left(\left(a_{0}(\xi, z) T\right.\right. \\
& \left.\left.+b_{0}(\xi, z)\right)^{m}\left(Z^{|\alpha|} g_{k}(\xi)\right)\right) \log \phi(\xi, z) d \xi_{1} \cdots d \xi_{n} d \bar{\xi}_{I}
\end{aligned}
$$

where $a_{0}(\xi, z)=-(T \phi)^{-1}$ and $b_{0}(\xi, z)=-T\left((T \phi)^{-1}\right)+h(T \phi)^{-1}$, and underline means there are $2^{|\alpha|-1}$ such terms. Again we see that both $a_{0}(\xi, z)$ and $b_{0}(\xi, z)$ are real analytic in $z$ and $\xi$ on $U_{z} \times U_{\xi}$. Now if we let $W$ be either $Z$ or $a_{0}(\xi, z) T+b_{0}(\xi, z)$, we get

$$
\frac{\partial^{\alpha}}{\partial z^{\alpha}} F_{1}(z)=\underline{2^{|\alpha|-1}} \cdot \frac{(-1)^{m}}{(m-1) !} \int_{b D}\left(W^{|\alpha|+m} g_{k}(\xi)\right) \log \phi(\xi, z) d \xi_{1} \cdots d \xi_{n} d \bar{\xi}_{I} .
$$

Next we observe that $g_{k}(\xi)$ can be viewed as a function defined on $(z, \xi)$ space, i.e., constant in $z$-variable. Hence if we let $X$ be either the vector field 
$T$ or $\frac{\partial}{\partial z_{j}}, j=1, \ldots, n$, then by estimate $(2.10)$ we obtain, for some constant $M>0$,

$$
\left|X^{j} g_{k}(\xi)\right| \leq M(M k)^{j}, \text { provided that } j \leq k .
$$

Also from the real analyticity of both $a_{l}(\xi, z)$ and $b_{l}(\xi, z)$ there exists a constant $M_{1}>0$ such that for all $(z, \xi) \in\left(V_{2}\right)_{z} \times\left(V_{2}\right)_{\xi}$ we have

$$
\left|D^{\beta} a_{l}(\xi, z)\right| \leq M_{1} M_{1}^{|\beta|} \cdot|\beta| !, l=0,1,2, \ldots, n,
$$

and

$$
\left|D^{\beta} b_{l}(\xi, z)\right| \leq M_{1} M_{1}^{|\beta|} \cdot|\beta| !, l=0,1,2, \ldots, n,
$$

where $D$ acts on both variables $z$ and $\xi$.

We claim that there exists a constant $S>0$ independent of $k,|\alpha|$ and $m$ such that

$$
\left|W^{|\alpha|+m} g_{k}(\xi)\right| \leq S(S k)^{|\alpha|+m},
$$

for all $(z, \xi) \in\left(V_{2}\right)_{z} \times\left(V_{2}\right)_{\xi}$, provided that $|\alpha|+m \leq k$.

Proof of the claim. By induction on $i$ we will show that

$$
\left|X^{j} W^{i} g_{k}\right| \leq M_{2} M_{3}^{j} M_{4}^{i} \cdot k^{j+i}
$$

holds for all $j$ for some constants $M_{2}, M_{3}$ and $M_{4}$, provided that $i+j \leq k$. This will prove our claim by setting $j=0$ and $i=|\alpha|+m$.

First we see that the initial step $i=0$ follows easily from the construction of $g_{k}$, i.e., estimate $(2.10)$ or (2.16). So we assume that (2.20) is true for all $j$ and $i<i_{0}$. Then we prove the case $i=i_{0}$ and all $j$. Put $i_{0}=i+1$ and $j+i+1 \leq k$.

Case 1. If $W^{i+1}=\frac{\partial}{\partial z_{l}} W^{i}$, for some $l \in\{1, \ldots, n\}$, we have

$$
\begin{aligned}
X^{j} W^{i+1} g_{k} & =X^{j} \frac{\partial}{\partial z_{l}} W^{i} g_{k} \\
& =X^{j+1} W^{i} g_{k} .
\end{aligned}
$$

Hence by induction hypothesis we get

$$
\begin{aligned}
\left|X^{j} W^{i+1} g_{k}\right| & \leq M_{2} M_{3}^{j+1} M_{4}^{i} \cdot k^{i+j+1} \\
& \leq M_{2} M_{3}^{j} M_{4}^{i+1} \cdot k^{j+i+1},
\end{aligned}
$$

provided that we choose $M_{4} \geq M_{3}$.

Case 2. If $W^{i+1}=\left(a_{l}(\xi, z) T+b_{l}(\xi, z)\right) W^{i}$ for some $l=0,1, \ldots, n$, we get

$$
\begin{aligned}
X^{j} W^{i_{0}} g_{k} & =X^{j}\left(a_{l}(\xi, z) T+b_{l}(\xi, z)\right) W^{i} g_{k} \\
& = \pm \sum_{p=0}^{j}(\underline{j})\left(X^{p} a_{l}\right) X^{j-p+1} W^{i} g_{k} \pm \sum_{p=0}^{j}(\underline{j})\left(X^{p} b_{l}\right) X^{j-p} W^{i} g_{k} \\
& =I+I I .
\end{aligned}
$$


Here underline again means that there are $(p)$ terms of the indicated form. Hence by induction hypothesis we obtain

$$
\begin{aligned}
|I| & \leq \sum_{p=0}^{j} \frac{j !}{p !(j-p) !} M_{1} M_{1}^{p} \cdot p ! \cdot M_{2} M_{3}^{j-p+1} \cdot M_{4}^{i} \cdot k^{i+j-p+1} \\
& =M_{2} M_{3}^{j} M_{4}^{i+1} \cdot k^{i+j+1} \sum_{p=0}^{j} \frac{j !}{(j-p) !} \cdot \frac{1}{k^{p}} \cdot \frac{M_{1} M_{1}^{p}}{M_{4} M_{3}^{p-1}} \\
& \leq M_{2} M_{3}^{j} M_{4}^{i+1} \cdot k^{i+j+1} \cdot\left(\frac{M_{1} M_{3}}{M_{4}}+\sum_{p=1}^{j} \frac{M_{1}^{2}}{M_{4}}\left(\frac{M_{1}}{M_{3}}\right)^{p-1}\right) \\
& \leq \frac{1}{2} M_{2} M_{3}^{j} M_{4}^{i_{0}} \cdot k^{j+i_{0}}
\end{aligned}
$$

provided that $M_{3} \geq 2 M_{1}$ and $M_{4} \geq \max \left(8 M_{1}^{2}, 4 M_{1} M_{3}\right)$.

$$
\begin{aligned}
|I I| & \leq \sum_{p=0}^{j} \frac{j !}{p !(j-p) !} M_{1} M_{1}^{p} \cdot p ! \cdot M_{2} M_{3}^{j-p} M_{4}^{i} \cdot k^{i+j-p} \\
& =M_{2} M_{3}^{j} M_{4}^{i+1} \cdot k^{i+j+1} \cdot \sum_{p=0}^{j} \frac{j !}{(j-p) !} \cdot \frac{1}{k^{p+1}} \cdot \frac{M_{1}}{M_{4}}\left(\frac{M_{1}}{M_{3}}\right)^{p} \\
& \leq M_{2} M_{3}^{j} M_{4}^{i+1} \cdot k^{i+j+1} \sum_{p=0}^{j}\left(\frac{M_{1}}{M_{4}}\right)\left(\frac{M_{1}}{M_{3}}\right)^{p} \\
& \leq \frac{1}{2} M_{2} M_{3}^{j} M_{4}^{i_{0}} \cdot k^{j+i_{0}}
\end{aligned}
$$

provided that $M_{3} \geq 2 M_{1}$ and $M_{4} \geq 4 M_{1}$.

This completes the proof of (2.20), and hence our claim (2.19).

So now if we combine the formula (2.15) and Lemma 2.4, let $k=|\alpha|+m$, we see that there exists a constant $S>0$ such that the following estimate holds uniformly for all $z \in D \cap B\left(z_{0}, \varepsilon\right)$ with $0<\varepsilon \ll \varepsilon_{1}$,

$$
\begin{aligned}
\left|\frac{\partial^{\alpha}}{\partial z^{\alpha}} F_{1}(z)\right| & \leq S(S(|\alpha|+m))^{|\alpha|+m} \\
& \leq S(3 S)^{|\alpha|+m} \cdot(|\alpha|+m) ! \\
& \leq S(3 S)^{|\alpha|+m} \cdot\left(2^{m}\right)^{|\alpha|+m} \cdot|\alpha| ! \\
& =S\left(3 \cdot 2^{m} \cdot S\right)^{m} \cdot\left(3 \cdot 2^{m} \cdot S\right)^{|\alpha|} \cdot|\alpha| ! \\
& \leq C C^{|\alpha|}|\alpha| !,
\end{aligned}
$$

provided that we choose $C=\max \left(3 \cdot 2^{m} \cdot S, S\left(3 \cdot 2^{m} \cdot S\right)^{m}\right)$.

This completes the proofs of Theorem 2.5 and Theorem 1. 


\section{ACKNOWLEDGMENT}

Finally the author would like to thank R. M. Range for fruitful discussions during the preparations of this paper.

\section{REFERENCES}

1. R. M. Range, The Carathéodory metric and holomorphic maps on a class of weakly pseudoconvex domains, Pacific J. Math. 78 (1978), 173-188.

2. Holomorphic functions and integral representations in several complex variables, Springer-Verlag, New York, 1986.

3. D. S. Tartakoff, On the global real analyticity of solutions to $\square_{b}$ on compact manifolds, Comm. in P.D.E., 1 (4) (1976), 283-311.

Department of Mathematics, State University of New York at Albany, Albany, NEW YORK 12222 